\title{
GaN hemispherical micro-cavities
}

\author{
Yiyun Zhang, ${ }^{1}$ Cong Feng, ${ }^{1}$ T. Wang, ${ }^{2}$ and H. W. Choi ${ }^{1, a)}$ \\ ${ }^{1}$ Department of Electrical and Electronic Engineering, The University of Hong Kong, Pokfulam Road, \\ Hong Kong \\ ${ }^{2}$ Department of Electronic and Electrical Engineering, University of Sheffield, Mappin Street, \\ Sheffield S1 3JD, United Kingdom
}

(Received 7 October 2015; accepted 9 January 2016; published online 21 January 2016)

\begin{abstract}
GaN-based micro-dome optical cavities supported on Si pedestals have been demonstrated by dry etching through gradually shrinking microspheres followed by wet-etch undercutting. Optically pumped whispering-gallery modes (WGMs) have been observed in the near-ultraviolet within the mushroom-like cavities, which do not support Fabry-Pérot resonances. The WGMs blue-shift monotonously as the excitation energies are around the lasing threshold. Concurrently, the modehopping effect is observed as the gain spectrum red-shifts under higher excitations. As the excitation energy density exceeds $\sim 15.1 \mathrm{~mJ} / \mathrm{cm}^{2}$, amplified spontaneous emission followed by optical lasing is attained at room temperature, evident from a super-linear increase in emission intensity together with linewidth reduction to $\sim 0.7 \mathrm{~nm}$ for the dominant WGM. Optical behaviors within these WGM microcavities are further investigated using numerical computations and three-dimensional finite-difference time-domain simulations. (C) 2016 AIP Publishing LLC.
\end{abstract}

[http://dx.doi.org/10.1063/1.4940375]

GaN-based microcavities are vital towards achieving high quality-factor ( $Q$-factor) lasing as well as being excellent platforms for investigating light-matter interactions in the strong-coupling regime. ${ }^{1-3} \mathrm{Up}$ to now, GaN optical microcavities of various geometrics have been studied, which generally include Fabry-Pérot $(F-P)$ cavities employing distributed Bragg reflectors (DBRs), ${ }^{4}$ defective photoniccrystal (PC) structures, ${ }^{5}$ and whispering-gallery mode (WGM) microcavities. ${ }^{6}$ Amongst these options, WGM microcavities have been demonstrated as promising candidates for realizing low-threshold lasing with narrow emission linewidths, attributed to their low optical losses and modal volumes. ${ }^{7,8}$ However, the superior optical confinement capabilities of GaN WGM microcavities are invariably weakened by optical leakage through its transparent sapphire substrate. Recently, GaN-based undercut two-dimensional (2D) WGM microdisk lasers fabricated through the removal of sacrificial layers embedded within the device structure by photoelectrochemical (PEC) or selective wet-etching ${ }^{9-11}$ have been reported to effectively reduce such losses. Similar undercut microdisk structures have also been demonstrated by controlled wet-etch removal of the underlying Si substrate using GaN-on-Si materials. ${ }^{12,13}$ There also are many other studies on GaN WGM microcavities based on self-assembled micro/ nano crystals using bottom-up methods, including microscale pyramids, hexagonal disks, and pillars. ${ }^{14-16}$ However, it is difficult to achieve precise control over the locations, morphologies, dimensions, and uniformities of these microstructures during growth. ${ }^{17}$ More importantly, in optical microcavities containing pairs of parallel mirror-like crystal facets, $F-P$ modes will always coexist with WGMs. ${ }^{16,18,19}$ On the contrary, optical microcavities of spherical or hemispherical geometries are ideal platforms for WGMs owing to

\footnotetext{
${ }^{\text {a) }}$ Author to whom correspondence should be addressed. Electronic mail: hwchoi@hku.hk. Tel.: (852) 28592693. Fax: (852) 25598738
}

outstanding three-dimensional (3D) optical confinement. ${ }^{20}$ Spherical or hemispherical microcavities are typically fabricated by the melting of silica glass, ${ }^{21,22}$ using liquid microdroplets levitated in air or self-assembled polymer hemispherical cavities on hydrophobic DBRs through surface tension. ${ }^{23-26}$ Although lasing actions have been demonstrated from these spherical/hemispherical cavities through addition of quantum dots or organic dyes as gain media, ${ }^{27}$ the control and maintenance over the dimensions and stabilities of such microcavities remain challenging. In this study, GaN undercut micro-dome 3D WGM optical cavities with embedded InGaN/GaN quantum wells (QWs) have been realized using microsphere lithography (MSL) and a combination of dry/wet etching. The optical characteristics of WGMs within these 3D micro-dome optical cavities are investigated by photoluminescence (PL) and 3D finitedifference time-domain (3D-FDTD) simulations. By eliminating $F$ - $P$ vertical modes, stimulated emission via WGM at ultraviolet (UV) wavelengths has been observed from these hemispheres.

Fig. 1(a) depicts the process flow for the fabrication of the micro-dome WGM resonators. Details about the epistructure of the GaN-on-Si wafer can be found elsewhere. ${ }^{13,28}$ First, silica microspheres with nominal diameters of $2-\mu \mathrm{m}$ are dispersed on the sample by spin-coating, utilizing a recipe that ensures that the microspheres are sparsely distributed across the wafer. Silica microspheres are used owing to their high thermal stabilities as well as sphericities, which are important attributes for high-definition pattern transfer. The sample is then etched using $\mathrm{Ar} / \mathrm{BCl}_{3} / \mathrm{SF}_{6}$ gases (10 sccm/10 sccm/25 sccm) by inductively coupled plasma (ICP) etching to form $\mathrm{GaN}$ micro-domes, using process conditions of $450 \mathrm{~W} / 75 \mathrm{~W}$ of coil/ platen powers at a chamber pressure of 5 mTorr. $\mathrm{SF}_{6}$ gas is added to increase the etch rate of the silica microspheres so that their lateral dimensions reduce as the etch proceeds; such gradual reduction of 
(a) $\mathrm{SiO}_{2}$ microspheres

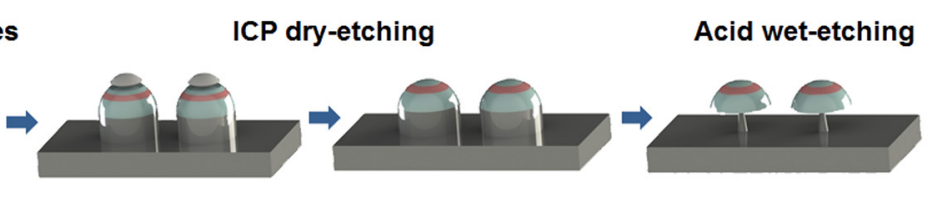

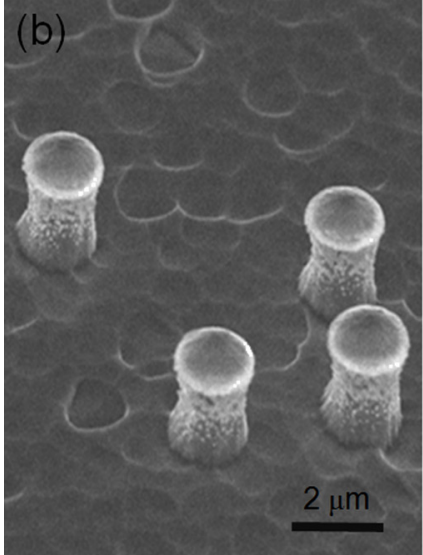
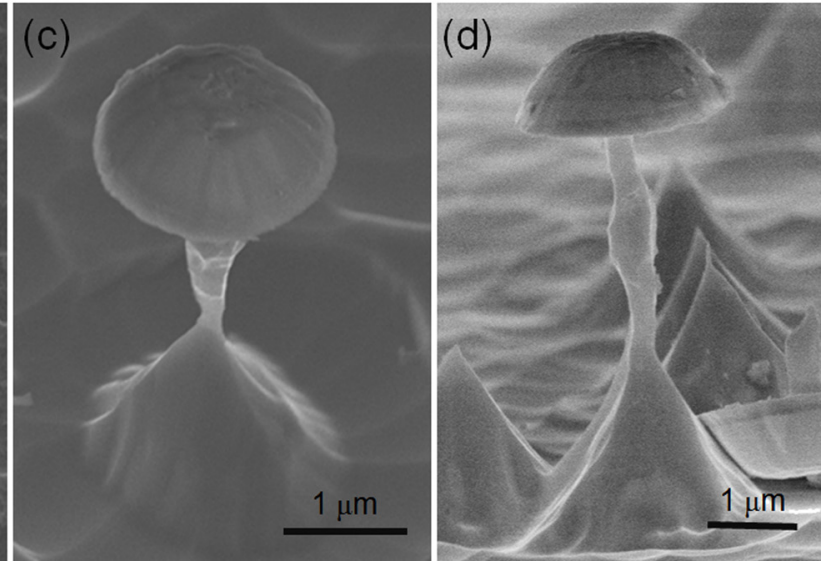

FIG. 1. (a) Schematic diagrams depicting the fabrication process flow of the $2-\mu \mathrm{m}$ GaN micro-dome suspended on Si. (b) FE-SEM images of GaN microdomes with $\mathrm{Si}$ bases before wetetching. FE-SEM images showing (c) bird's eye and (d) cross-sectional views of a single GaN micro-dome suspended on a $\mathrm{Si}$ post after wetetching. diameter results in the formation of micro-domes in the GaN layer. The field-emission scanning electronic microscope (FE-SEM) image in Fig. 1(b) shows the GaN micro-domes with average diameters of $2-\mu \mathrm{m}$ on top Si pedestals after the ICP dry-etching, which terminates approximately $5 \mu \mathrm{m}$ beneath the GaN-Si interface. After this, the sample is immersed into an $\mathrm{HF} / \mathrm{HNO}_{3}$ (1:1) acid solution for $1 \mathrm{~min}$ which etches the $\mathrm{Si}$ beneath the micro-domes, creating $\mathrm{Si}$ nano-posts with diameters of about $\sim 100 \mathrm{~nm}$ that mechanically support the overhanging GaN micro-domes. This wet process needs to be precisely controlled so that the Si nanoposts will not be completely etched off, preventing the collapse of the micro-domes. Bird's eye view and crosssectional view of a single $\mathrm{GaN}$ micro-dome suspended atop a Si post are presented in Figs. 1(c) and 1(d). The roomtemperature (RT) $\mu$-PL measurements are conducted using a diode-pumped solid state (DPSS) pulsed laser emitting at $349 \mathrm{~nm}$ as an excitation source, with pulse durations of $4 \mathrm{ns,}$ repetition rates of $1 \mathrm{kHz}$, and maximum pulse energies of $120 \mu \mathrm{J}$. The PL signals are probed by a multi-mode optical fiber placed in close proximity to the sample and coupled to an optical spectrometer with optical resolution of $\sim 0.04 \mathrm{~nm}$. The position of the fiber probe is fixed after the optimal optical coupling has been achieved, pointing to the micro-dome structures at an angle of $\sim 15^{\circ}$ to the horizontal plane.

Fig. 2(a) shows the RT- $\mu$ PL spectra of the GaN microdomes with increasing excitation energy densities. At low excitation energies, two dominant broadband peaks centered at $\sim 370 \mathrm{~nm}$ and $\sim 450 \mathrm{~nm}$ can be clearly identified from the PL spectra, corresponding to emissions from GaN materials and InGaN/GaN MQWs, respectively. The PL intensities of the blue emission band from the InGaN/GaN QWs are much weaker than that from GaN, as shown in the inset of Fig. 2(a), due to partial removal of the QWs during dry etching, retaining a small portion embedded at the top of microdomes. This can be overcome by growing a thicker cap layer over the QWs. As the excitation energy densities are further increased to above $\sim 11.8 \mathrm{~mJ} / \mathrm{cm}^{2}$, several additional narrow spectral peaks centered at $\sim 371.6 \mathrm{~nm}, \sim 376.4 \mathrm{~nm}$, $\sim 381.4 \mathrm{~nm}$, and $\sim 386.8 \mathrm{~nm}$ emerge in the near-UV range, labeled as $P_{1,2,3,4}$ in Fig. 2(a). Here, it is worthy of noting that these peaks only appear at the low-energy side of the PL spectra, attributed to stronger absorption in the gain mediums at shorter wavelengths due to the reduced Stokes shift between absorption and emission. ${ }^{29}$ Furthermore, the
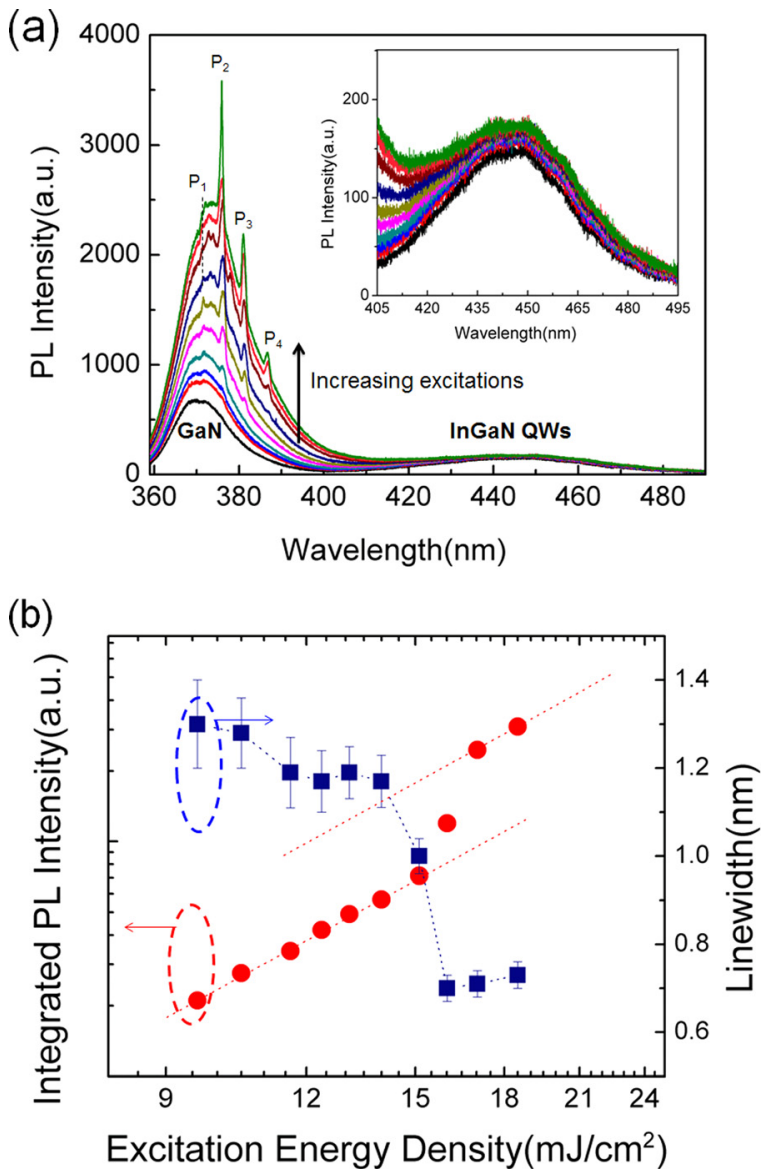

FIG. 2. (a) RT- $\mu \mathrm{PL}$ spectra from GaN micro-domes with increasing laser excitations. The inset shows an enlarged view of emissions from the InGaN/ GaN QWs. (b) Log-log plot of integrated PL intensities against excitation powers and linewidth evolution of the lasing peak at $\sim 376 \mathrm{~nm}$. 
non-observation of optical modes in the QWs emission range, even under very high excitation levels as plotted in the inset of Fig. 2(a), can be attributed to the low gains of the partially removed QWs, as well as poor coupling to the strong fundamental WGMs. As a matter of fact, the undercut beneath the micro-dome will minimize optical absorptions of the Si substrate by reflecting light at the semiconductor-air interface, promoting optical confinement in the vertical direction. However, the curvature of the micro-dome will divert these rays into non-vertical directions for couplings into WGMs at the boundary of the domes, whilst preventing wave-guide resonances coupling into stable $F-P$ vertical modes. As a result, this mushroom-like structure will facilitate lateral optical confinement into WGMs at the boundary of the micro-dome resonators, while suppressing the formation of stand-waves in the vertical direction.

The log-log plot of integrated PL intensities and spectral linewidth of the WGM at $\sim 376 \mathrm{~nm}$ as functions of excitation power densities is shown in Fig. 2(b). When the excitations are low, the peak integrated PL intensities will increase linearly with increasing the excitations, attributed to the spontaneous emission of $\mathrm{GaN}$ micro-domes. A nonlinear increase in the integrated PL intensities is observed as the excitation power exceeds $\sim 15.1 \mathrm{~mJ} / \mathrm{cm}^{2}\left(\mathrm{E}_{t h}\right)$. This superlinear increase is a clear sign that amplified spontaneous emission (ASE) has been achieved, demonstrating a transition process of spontaneous emission to stimulated emission within these micro-domes. Since positive feedback of the ASE can further generate lasing if the optical gain surpasses the losses via optical coupling with WGMs, the emergence of the sharp spectral peaks indicates that near-UV lasing actions have been established within these micro-dome WG cavities. This is also demonstrated by the saturation in the integrated PL intensities at $\sim 1.2 \mathrm{E}_{t h}$. Meanwhile, the spectral linewidth of the WGM at $\sim 376 \mathrm{~nm}$ narrows from $\sim 1.2 \mathrm{~nm}$ at $\sim 0.8 \mathrm{E}_{t h}$ to $\sim 0.7 \mathrm{~nm}$ at $\sim 1.1 \mathrm{E}_{t h}$, further confirming that optical absorption has been bleached and that the lasing regime has been attained. A $Q$-factor of $\sim 530$ at the transparency can be roughly estimated using the relation $Q=\lambda / \delta \lambda$, where $\lambda$ and $\delta \lambda$ represent the wavelength and spectral linewidth, respectively. Improvements to the $Q$ factor can be achieved by refining the surface roughness during the ICP dry-etching or chemical wet-etching processes. ${ }^{2,30}$ And the spontaneous emission coupling factor $(\beta)$ can be roughly estimated to be $\sim 0.28$, taking the ratio of PL intensities below and above the threshold. Moreover, as the excitation energy densities gradually increase from $\sim 0.6 \mathrm{E}_{t h}$ to $\sim 1.1 \mathrm{E}_{t h}$, a continuous blueshift in the peak wavelengths of the observed WGMs $\left(P_{1,2,3,4}\right)$ can be identified. Fig. 3(a) shows a magnified view of the PL spectra around $P_{3}$ under different excitation levels, from which a clear blue-shift from $381.4 \mathrm{~nm}$ to $381.0 \mathrm{~nm}$ can be distinguished. In addition, it can be found that all WGM wavelengths blue-shift linearly as functions of excitation powers in the range of $\sim 0.6 \mathrm{E}_{t h}$ to $\sim 1.1 \mathrm{E}_{t h}$, as shown in Fig. 3 (b). Since the existence of WGMs reflect the satisfaction of standing-wave conditions $\left(\mathrm{m} \lambda=2 \pi R n_{\text {eff }}\right)$ within the cavities, the shifts of WGM wavelengths are thereafter determined by the fluctuations $(\Delta n)$ of the effective refraction index $\left(n_{\text {eff }}\right)$. As a matter of fact, $\Delta n$ reflects the change of optical absorption coefficient, which can be strongly influenced by a (a)
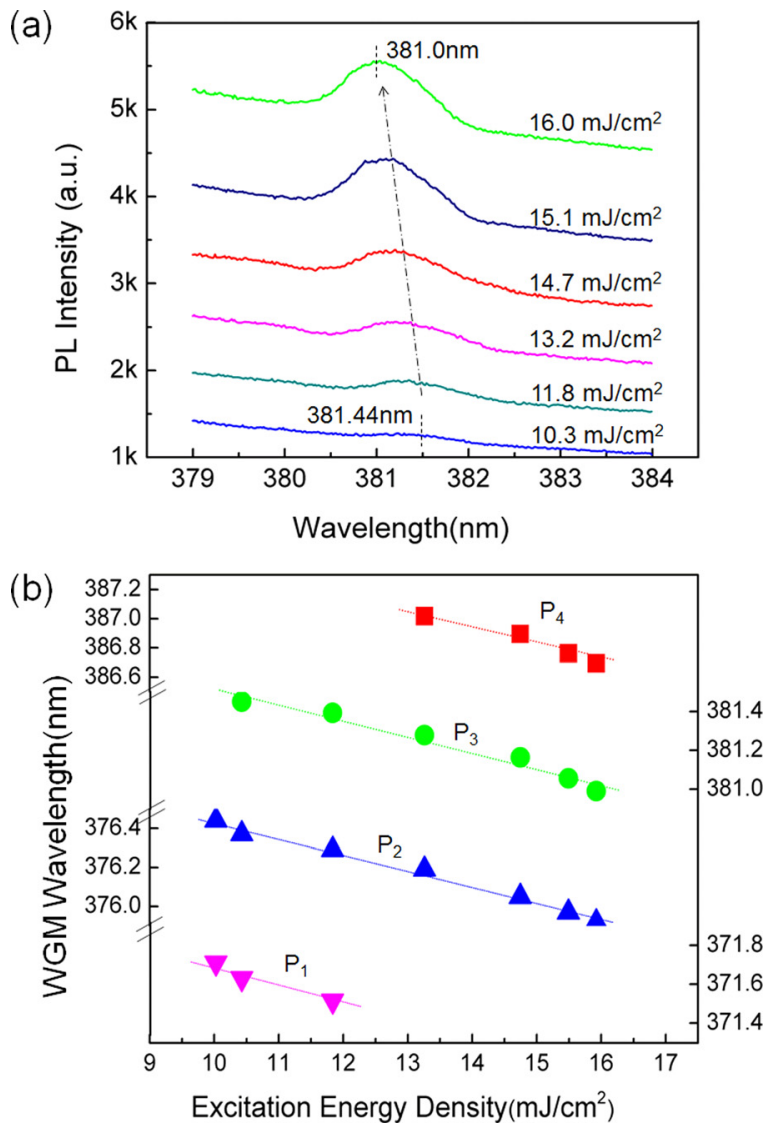

FIG. 3. (a) $\mu$-PL spectra in the vicinity of the $P_{3}$ mode under different excitation energy densities. For clarity, the spectra have been vertically offset. (b) Blue-shifts in wavelengths of the observed WGMs $\left(P_{1,2,3,4}\right)$ with increasing excitation energy densities.

combination of three major factors: carrier band-filling, energy bandgap shrinkage, and plasma effects. ${ }^{31-33}$ At subthreshold conditions whereby the lowest energy states near the band edge have been filled up, more energy is needed for electrons to be excited to the higher energy states within the conduction band from the valence band. This band-filling effect, together with the absorption of free carriers, will lead to a reduction of the absorption coefficient, which results in a negative $\Delta n$ for wavelengths near or below the energy band-gap. ${ }^{33}$ This carrier-induced negative $\Delta n$ may explain the linear blue-shift phenomenon of the WGMs with increasing carrier concentrations (excitation). Thereafter, as the excitation levels are further increased far beyond the threshold, band-gap shrinkage caused by renormalization (electronelectron and electron-ion interactions) and heating effects (non-radiative recombination) will become dominant and give rise to a positive $\Delta n$ as the absorption coefficient increases, potentially resulting in red-shifts of the WGM wavelengths. This phenomenon has already been observed in GaN-on-Si micro-disk cavities before, in which electronhole plasma (EHP) effects are likely to contribute concurrently. ${ }^{13}$ Although thermal effects are not dominant to WGMs in the micro-domes, the broadband PL gain spectrum is influenced by heat accumulation as the Si pivots are the main thermal conduction pathways, resulting in red-shifts of peak wavelengths from $\sim 370.6 \mathrm{~nm}$ to $\sim 373.8 \mathrm{~nm}$ as the excitation increased from $\sim 0.6 \mathrm{E}_{t h}$ to $\sim 1.1 \mathrm{E}_{t h}$. Consequently, mode hopping occurs as the gain red-shifts, as illustrated in 
Fig. 4. At $\sim 0.8 \mathrm{E}_{t h}$, three spectral peaks $\left(P_{1}, P_{2}, P_{3}\right)$ appear in the PL spectrum. When the excitation is increased to $\sim 1.1 \mathrm{E}_{t h}$, as the gain red-shifts to the lower energy side, the WGM at shorter wavelength $\left(P_{1}\right)$ gradually disappears while $P_{4}$ shows up on the lower energy side.

Optical behaviors of the WGMs within a micro-dome cavity are further investigated by numerical calculations and 3D-FDTD simulations. A model of the micro-dome cavity in spherical coordinates is shown in Fig. 5(a). The radius of the micro-dome and the $\mathrm{n}_{\text {eff }}$ of $\mathrm{GaN}$ materials are set to be $\sim 1.0 \mu \mathrm{m}$ and 2.5 , respectively. The electric dipoles are randomly distributed in the micro-dome cavity, while two plane-monitors are placed along a cross-sectional plane and at the bottom of the micro-dome to detect the electromagnetic fields in the vertical and in-plane directions. The simulated optical resonances within the micro-dome cavity are displayed in Fig. 4, together with the mode numbers in the sequence of $(n, m, q)$ being labeled for these dominant fundamental and first-order optical modes, where $\mathrm{n}, \mathrm{m}$, q represent the mode numbers in the radial $(r)$, azimuthal $(\varphi)$, and polar angular $(\theta)$ direction, respectively. Additionally, transverse-electric polarized (TE) or transverse-magnetic polarized (TM) WGMs are defined as optical modes whose electric or magnetic fields are parallel to the hemispherical surface of the micro-domes. The simulation results show that only several orders of WGMs can be supported in the microdome cavity, while $F-P$ vertical modes are totally ruled out. All the experimentally detected spectral peaks $\left(P_{1,2,3,4}\right)$ are found to belong to the first mode family $(n=1)$ WGMs with different mode numbers in the lateral direction. Specifically, $P_{1}$ and $P_{3}$ are attributed to TE modes $(\mathrm{m}=38,37 ; \mathrm{q}=2)$, while $P_{2}$ and $P_{4}$ are fundamental order TM modes $(\mathrm{m}=37$, $36 ; \mathrm{q}=1)$. The in-plane and cross-sectional electric-field energy intensity patterns for the fundamental TM mode at $\sim 376 \mathrm{~nm}\left(P_{2}\right)$ and first-order TE patterns at $\sim 381 \mathrm{~nm}\left(P_{3}\right)$ are presented in Figs. 5(b)-5(e). Regions shaded in red and blue represent the highest and lowest field intensities, respectively. The electric-field distributions for these TE and TM

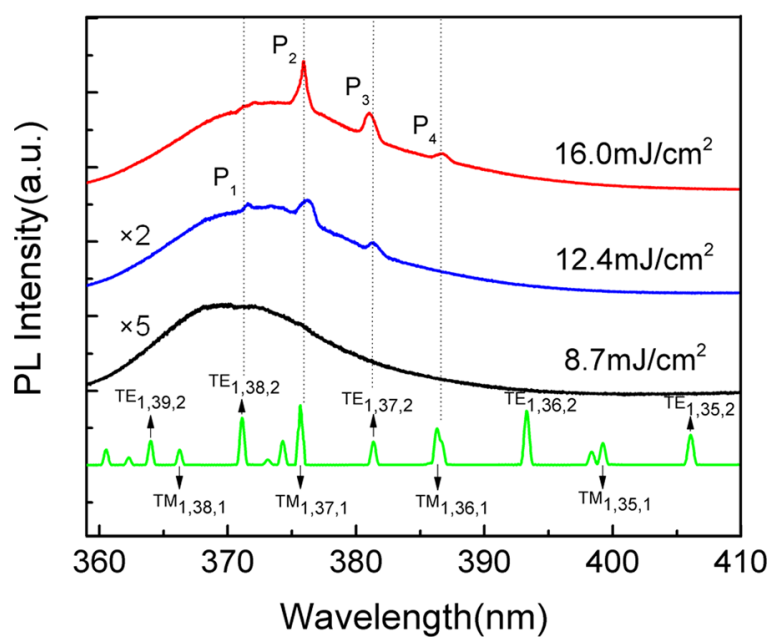

FIG. 4. The mode-hopping effect shown in the PL spectra with increasing excitation energy densities. The spectra have been vertically offset for clarity. The green plot at the bottom shows the possible optical resonances within a $2.06 \mu \mathrm{m}$ GaN hemispherical cavity as predicted by 3D-FDTD simulations. (a)
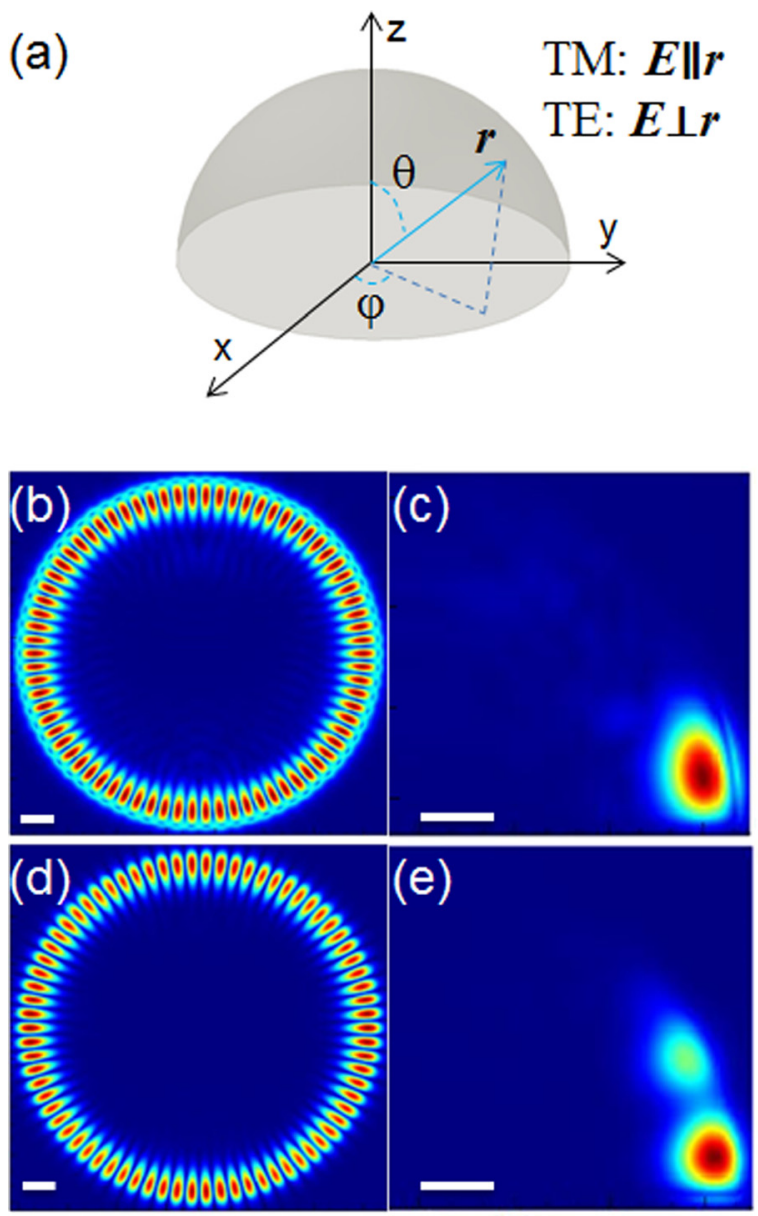

FIG. 5. (a) Modeling of the GaN micro-dome in hemispherical coordinates. The electric-field energy intensity patterns from 3D-FDTD simulations for fundamental transverse-electric (TM) mode at $\sim 376 \mathrm{~nm}$ in the (b) $\mathrm{x}-\mathrm{y}$ and (c) $\mathrm{X}-\mathrm{Z}$ planes, and for first-order transverse-magnetic (TE) mode at $\sim 381 \mathrm{~nm}$ in the (d) $\mathrm{x}-\mathrm{y}$ and (e) $\mathrm{x}-\mathrm{z}$ planes. Regions shaded in red and blue represent the highest and lowest field intensities, respectively. The scale bars represent lengths of $200 \mathrm{~nm}$.

modes can be found in Fig. S1. ${ }^{34}$ Although some high-order WGMs can coexist within the micro-dome cavity according to the simulations, they are not appearing in the experimental PL spectra, as these high-order modes always possess larger mode volumes that can induce higher optical losses due to re-absorption effect. ${ }^{35}$ It is also possible that these highorder WGMs have not been picked up by the fiber probe, whose orientation with respect to the micro-domes is fixed. Afterwards, the free spectral range (FSR) is further analyzed using 2D numerical computations, since the WGMs are actually confined close to the bottom edge of the microdome cavity. ${ }^{25,26}$ Derived from solving the 2D Helmholtz equation via separation of variables, the mode spacing $(\Delta \lambda)$ between adjacent modes can be roughly calculated by ${ }^{6}$

$$
\Delta \lambda=\frac{\lambda_{w g m}^{2}}{2 \pi R\left(n_{e f f}-\lambda_{w g m} \frac{d n}{d \lambda}\right)},
$$

where $R$ represents the radius of the micro-dome, $\lambda_{w g m}$ is the WGM wavelengths, and $d n / d \lambda$ is the first-order dispersion in GaN. Using the Sellmeier equation for GaN, we have obtained theoretical values of $\sim 9.3 \mathrm{~nm}$ for TE modes 
$\left(P_{1}, P_{3}\right)$ and $\sim 9.9 \mathrm{~nm}$ for TM modes $\left(P_{2}, P_{4}\right)$, which are nearly consistent with the spectral spacing of $9.8 \mathrm{~nm}$ and $10.4 \mathrm{~nm}$ evaluated from the PL spectrum, respectively. The relatively lower value is attributed to the use of the geometrical radius in the calculations, which will be slightly larger than the effective radius of the micro-dome cavity for the optical modes. These simulation results also suggest that the QWs should be located close to bottom plane of the hemispherical microcavities to achieve optimal optical coupling between WGMs and the gain spectrum; of course growing high-quality QWs close to the substrate is challenging.

In summary, $2-\mu \mathrm{m} \mathrm{GaN}$ undercut micro-dome WG resonators on Si substrates have been demonstrated using a combination of MSL and wet/ dry etch processes. As vertical $F$ $P$ modes are not supported by the hemispherical cavity, the observation of optically pumped stimulated emission can affirmatively be attributed to WGMs. Room-temperature lasing via WGMs at near-UV wavelengths with narrow linewidths of $\sim 0.7 \mathrm{~nm}$ and $Q$-factors exceeding $\sim 500$ has been achieved, enabled by 3D optical confinement within the micro-domes. Optical behaviors of WGMs within these cavities have been studied carefully, revealing that carrier dynamics play a significant role in determining WGM frequencies and mode competitions with increasing freecarrier concentrations under various levels of excitations. Numerical calculations and 3D-FDTD simulations have further been carried out to identify the modal family and to determine the mode patterns of the WGMs that are detectable from PL. This proof of concept demonstration paves the way towards 3D GaN microcavities and coherent light emitters which can potentially outperform its $2 \mathrm{D}$ counterparts.

This work was supported by the General Research Fund (Project Code 17201614) of the Research Grants Council of Hong Kong.

${ }^{1}$ T. Someya, R. Werner, A. Forchel, M. Catalano, R. Cingolani, and Y. Arakawa, Science 285, 1905 (1999).

${ }^{2}$ Y. Zhang, X. Zhang, K. H. Li, Y. F. Cheung, and H. W. Choi, Phys. Status Solidi A 212(5), 960 (2015).

${ }^{3}$ S. Christopoulos, G. Baldassarri Hoger von Hogersthal, A. J. D. Grundy, P. G. Lagoudakis, A. V. Kavokin, J. J. Baumberg, G. Christmann, R. Butte, E. Feltin, J.-F. Carlin, and N. Grandjean, Phys. Rev. Lett. 98, 126405 (2007).

${ }^{4}$ R. Chen, H. D. Sun, T. Wang, K. N. Hui, and H. W. Choi, Appl. Phys. Lett. 96, 241101 (2010).

${ }^{5}$ N. Vico Triviño, G. Rossbach, U. Dharanipathy, J. Levrat, A. Castiglia, J.F. Carlin, K. A. Atlasov, R. Butté, R. Houdré, and N. Grandjean, Appl. Phys. Lett. 100, 071103 (2012).
${ }^{6}$ M. Kneissl, M. Teepe, N. Miyashita, N. M. Johnson, G. D. Chern, and R. K. Chang, Appl. Phys. Lett. 84, 2485 (2004).

${ }^{7}$ I. Aharonovich, A. Woolf, K. J. Russell, T. Zhu, N. Niu, M. J. Kappers, R. A. Oliver, and E. L. Hu, Appl. Phys. Lett. 103, 021112 (2013).

${ }^{8}$ M. Mexis, S. Sergent, T. Guillet, C. Brimont, T. Bretagnon, B. Gil, F. Semond, M. Leroux, D. Néel, S. David, X. Chécoury, and P. Boucaud, Opt. Lett. 36(12), 2203 (2011).

${ }^{9}$ E. D. Haberer, R. Sharma, C. Meier, A. R. Stonas, S. Nakamura, S. P. DenBaars, and E. L. Hu, Appl. Phys. Lett. 85, 5179 (2004).

${ }^{10}$ A. C. Tamboli, E. D. Haberer, R. Sharma, K. H. Lee, S. Nakamura, and E. L. Hu, Nat. Photonics 1, 61 (2007).

${ }^{11}$ D. Simeonov, E. Feltin, A. Altoukhov, A. Castiglia, J.-F. Carlin, R. Butté, and N. Grandjean, Appl. Phys. Lett. 92, 171102 (2008).

${ }^{12}$ H. W. Choi, K. N. Hui, P. T. Lai, P. Chen, X. H. Zhang, S. Tripathy, J. H. Teng, and S. J. Chua, Appl. Phys. Lett. 89, 211101 (2006).

${ }^{13}$ Y. Zhang, Z. Ma, X. Zhang, T. Wang, and H. W. Choi, Appl. Phys. Lett. 104, 221106 (2014).

${ }^{14}$ H. X. Jiang, J. Y. Lin, K. C. Zeng, and W. Yang, Appl. Phys. Lett. 75, 763 (1999).

${ }^{15}$ T. Kouno, K. Kishino, and M. Sakai, IEEE J. Quantum Electron. 47(12), $1565(2011)$.

${ }^{16}$ P.-M. Coulon, M. Hugues, B. Alloing, E. Beraudo, M. Leroux, and J. Zuniga-Perez, Opt. Express 20(17), 18707 (2012).

${ }^{17} \mathrm{~S}$. Li and A. Waag, J. Appl. Phys. 111, 071101 (2012).

${ }^{18}$ H. Baek, J. K. Hyun, K. Chung, H. Oh, and G.-C. Yi, Appl. Phys. Lett. 105, 201108 (2014)

${ }^{19}$ J. C. Ahn, K. S. Kwak, B. H. Park, H. Y. Kang, J. Y. Kim, and O'Dae Kwon, Phys. Rev. Lett. 82, 536 (1999).

${ }^{20}$ K. J. Vahala, Nature 424, 839 (2003).

${ }^{21}$ A. Chiasera, Y. Dumeige, P. Féron, M. Ferrari, Y. Jestin, G. N. Conti, S. Pelli, S. Soria, and G. C. Righini, Laser Photonics Rev. 4, 457 (2010).

${ }^{22}$ G. Cui, J. M. Hannigan, R. Loeckenhoff, F. M. Matinaga, M. G. Raymer, S. Bhongale, M. Holland, S. Mosor, S. Chatterjee, H. M. Gibbs, and G. Khitrova, Opt. Express 14(6), 2289 (2006).

${ }^{23}$ S.-X. Qian, J. B. Snow, H.-M. Tzeng, and R. K. Chang, Science 231, 486 (1986).

${ }^{24}$ J. Schäfer, J. P. Mondia, R. Sharma, Z. H. Lu, A. S. Susha, A. L. Rogach, and L. J. Wang, Nano Lett. 8(6), 1709 (2008).

${ }^{25}$ J. Haase, S. Shinohara, P. Mundra, G. Risse, V. G. Lyssenko, H. Fröb, M. Hentschel, A. Eychmüller, and K. Leo, Appl. Phys. Lett. 97, 211101 (2010).

${ }^{26}$ V. D. Ta, R. Chen, and H. D. Sun, Adv. Mater. 24(10), OP60 (2012).

${ }^{27}$ R. Chen, V. D. Ta, and H. D. Sun, Sci. Rep. 2, 244 (2012).

${ }^{28}$ M. Athanasiou, R. Smith, B. Liu, and T. Wang, Sci. Rep. 4, 7250 (2014).

${ }^{29}$ D. Simeonov, E. Feltin, H.-J. Bühlmann, T. Zhu, A. Castiglia, M. Mosca, J.-F. Carlin, R. Butté, and N. Grandjean, Appl. Phys. Lett. 90, 061106 (2007).

${ }^{30}$ M. Kim, D. Kim, S. Lee, and O’Dae Kwon, J. Appl. Phys. 96, 4742 (2004).

${ }^{31}$ E. Kuokstis, J. W. Yang, G. Simin, M. Asif Khan, R. Gaska, and M. S. Shur, Appl. Phys. Lett. 80, 977 (2002).

${ }^{32}$ L.-H. Peng, C.-W. Chuang, and L.-H. Lou, Appl. Phys. Lett. 74, 795 (1999).

${ }^{33}$ B. R. Bennett, R. A. Soref, and J. A. Del Alamo, IEEE J. Quantum Electron. 26(1), 113 (1990).

${ }^{34}$ See supplementary material at http://dx.doi.org/10.1063/1.4940375 for electric-field distributions ( $\mathrm{x}-\mathrm{z}$ plane) of whispering-gallery modes in $\mathrm{GaN}$ hemispherical micro-cavities from 3D-FDTD simulations.

${ }^{35}$ A. B. Matsko and V. S. Ilchenko, IEEE J. Sel. Top. Quantum Electron. 12(1), 3 (2006). 